\title{
Tomographic retrieval of cloud liquid water fields from a single scanning microwave radiometer aboard a moving platform - Part 2: Observation system simulation experiments
}

\author{
D. Huang ${ }^{1}$, A. Gasiewski ${ }^{2}$, and W. Wiscombe ${ }^{1,3}$ \\ ${ }^{1}$ Brookhaven National Laboratory, Upton, NY 11973, USA \\ ${ }^{2}$ University of Colorado, Boulder, CO 80309, USA \\ ${ }^{3}$ NASA Goddard Space Flight Center (Code 913), Greenbelt, MD 20771, USA
}

Received: 14 April 2009 - Published in Atmos. Chem. Phys. Discuss.: 15 May 2009

Revised: 7 July 2010 - Accepted: 10 July 2010 - Published: 22 July 2010

\begin{abstract}
Part 1 of this research concluded that many conditions of the 2003 Wakasa Bay experiment were not optimal for the purpose of tomographic retrieval. Part 2 (this paper) then aims to find possible improvements to the mobile cloud tomography method using observation system simulation experiments. We demonstrate that the incorporation of the $L_{1}$ norm total variation regularization in the tomographic retrieval algorithm better reproduces discontinuous structures than the widely used $L_{2}$ norm Tikhonov regularization. The simulation experiments reveal that a typical ground-based mobile setup substantially outperforms an airborne one because the ground-based setup usually moves slower and has greater contrast in microwave brightness between clouds and the background. It is shown that, as expected, the error in the cloud tomography retrievals increases monotonically with both the radiometer noise level and the uncertainty in the estimate of background brightness temperature. It is also revealed that a lower speed of platform motion or a faster scanning radiometer results in more scan cycles and more overlap between the swaths of successive scan cycles, both of which help to improve the retrieval accuracy. The last factor examined is aircraft height. It is found that the optimal aircraft height is 0.5 to $1.0 \mathrm{~km}$ above the cloud top. To summarize, this research demonstrates the feasibility of tomographically retrieving the spatial structure of cloud liquid
\end{abstract}

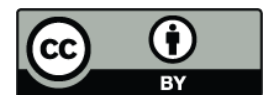

Correspondence to: D. Huang (dhuang@bnl.gov) water using current microwave radiometric technology and provides several general guidelines to improve future fieldbased studies of cloud tomography.

\section{Introduction}

Clouds in the lower troposphere exert large influences on the Earth's radiation budget and also play a crucial role in the planet's hydrological cycle. The spatial distribution of cloud water is one of the most poorly represented components in numerical models and is considered as one of the largest uncertainties in climate change predictions (Weare, 1996; Stephens, 2005). Part of the reason is that the scarcity of consistent long-term cloud observations makes it difficult to test and evaluate the cloud representations in the models. In order to enhance cloud process studies and to improve cloud representations, we need observational techniques that are capable of providing accurate cloud observations for a relatively large domain at suitable temporal and spatial resolution. For example, the validation of large eddy simulation models requires cloud observations at a temporal resolution of a few seconds and a spatial resolution of a few tens of meters over a domain of a few tens of kilometers. Aircraft-based in-situ measurements can sample only a small volume of a cloud, while the widely-used vertically-pointing microwave radiometers, such as those used at the Department of Energy Atmospheric Radiation Measurement sites, are capable of providing only the vertically integrated Liquid

Published by Copernicus Publications on behalf of the European Geosciences Union. 
Water Content (LWC) (Liljegren et al., 2001; Westwater et al., 2004). On the other hand, active remote sensing techniques like cloud radar (Frisch et al., 1995; Hogan et al., 2005) with rapid scanning capability provide a less direct measurement of cloud LWC (since LWC is proportional to the third moment of cloud drop size distribution but radar reflectivity is proportional to the sixth moment) and also would likely be much more costly than passive methods.

Tomographic methods provide great potential to address the cloud observation problem. It was first proposed in the 1980s that the microwave cloud tomography method can be used to retrieve three-dimensional cloud LWC by probing the thermal emission of clouds using either multiple distinctlylocated ground-based microwave radiometers or a single radiometer deployed on a mobile platform. The reconstruction of cloud water spatial distributions from the tomographic observations can be converted into a matrix inversion problem (Warner et al., 1985; Drake and Warner, 1988; and Warner and Drake, 1988). The fixed ground-based configuration has the apparent advantage of continuously operation without human intervention and thus it is well suited for long-term deployment. It was revealed recently that the retrieval accuracy of the fixed ground-based configuration depends on the number of microwave radiometers as well as their physical arrangement (Huang et al., 2008a). At least four radiometers are needed for the fixed configuration to obtain a cloud reconstruction that is accurate to within $10 \%$ of the cloud's maximum LWC. The requirement for a large number of microwave radiometers makes the fixed tomography configuration expensive to deploy. Furthermore, the fixed configuration has no cloud chasing capability and thus cannot be used to study the full cloud lifecycle (clouds can move out of the radiometer's field of view in a few minutes).

Alternatively, a mobile tomography configuration deployed on a moving platform needs only one scanning radiometer to collect tomographic data of similar quality. Geometrically, tomographic reconstruction requires the object (it is clouds for cloud tomography) to be scanned from multiple locations and directions. In other words, two scanning mechanisms are needed: spatial scanning and angular scanning. The spatial scanning of clouds is achieved in a mobile setup by the horizontal motion of the platform. A first investigation of this configuration was performed by Drake and Warner (1988), in which the radiometer switches automatically between two fixed antennas as the platform moves along a horizontal line passing just under a cloud. They showed that the dual-antenna setup performed similarly to a fixed ground-based setup using two scanning radiometers. A follow-up field test was conducted in Louisiana, and the LWC deduced from the radiometric measurements showed statistically good agreement with that measured directly by a Particle Measuring System version of liquid water probe but a point-by-point comparison was not made due to a scale mismatch between the two techniques (Warner and Drake, 1988).
It was shown that one of the most important factors for cloud tomography observations is the number of directions from which each cloud pixel is viewed (Huang et al., 2008a). The use of a dual-antenna radiometer in Warner's setup (strictly speaking, it is not a scanning radiometer) allows for only two viewing directions, which is far from optimal and would result in insufficient information for retrieving cloud liquid water structures (Huang et al., 2008a). The angular scanning of clouds can be improved by replacing the dualantenna radiometer with a scanning radiometer that can scan continuously at different elevation and azimuth angles.

A very limited cloud tomography experiment was conducted during the 2003 AMSR-E validation campaign at Wakasa Bay of the Sea of Japan (Lobl et al., 2007), where the Polarimetric Scanning Radiometer (Piepmeier and Gasiewski, 1996) was mounted to the bottom of the NASA P-3 research aircraft and scanned through a system of lowaltitude clouds from above. In a companion paper (Huang et al., 2010, hereinafter referred to as Part 1) we demonstrated that, although the tomographic retrievals from the Wakasa Bay experiment roughly capture the spatial features of clouds compared to a radar image, they fail to reproduce high-frequency structures. It was found that many conditions of the Wakasa Bay experiment were not optimal for cloud tomography retrieval, such as strong surface wind and high speed of aircraft motion. A more thorough examination of the mobile tomography method is thus needed in order to determine the optimal configuration and data collection strategy. These are the focus of Part 2 (this paper) of this research.

Two possible mobile configurations are considered in this paper: a downward-looking airborne configuration and an upward-looking ground-based configuration (Fig. 1). The airborne configuration was used in the Wakasa Bay cloud tomography experiment. The microwave radiometer is mounted to the bottom of the aircraft, which is a widely-used design of high-altitude research aircraft. With this design, the radiometer has to look downward in order to observe the cloud microwave emission. Figure 1 shows the swaths of three successive scan cycles, each labeled in a different color. The aircraft translates a certain distance along the flight track during each scan cycle, and the location of the aircraft for each cycle is also shown in the figure. The other configuration shown in Fig. 1 is a ground-based cloud tomography system that uses a pickup truck as the platform. With this configuration, clouds can only be scanned from below. Four scan cycles are shown in Fig. 1 for the this configuration, suggesting that the slower ground-based platform usually allows for more scan cycles than its airborne counterpart. More details on the difference between the ground-based and airborne configurations and their relevance to cloud tomography retrieval are described in Sect. 4.

The paper is organized as follows. In Sect. 2 we present the details of the observation system simulation experiments for cloud tomography. Section 3 examines the skill of two different retrieval algorithms: one based on the standard 


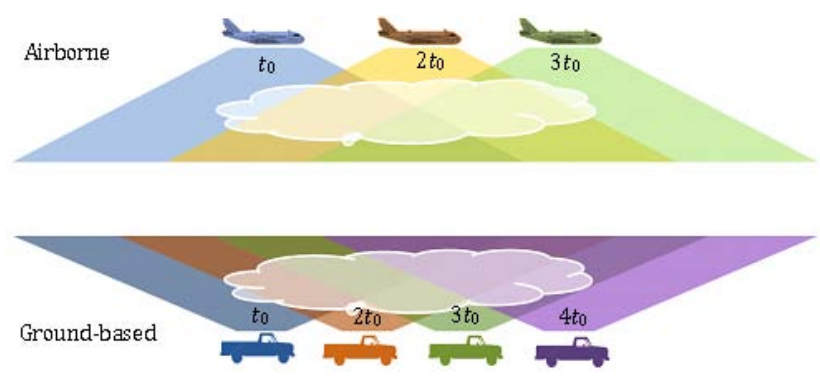

Fig. 1. Illustration of the observation geometry of the ground-based and airborne tomography configurations. The cartoon shows the swaths of radiometer scan at time $t=t_{0}, 2 t_{0}, 3 t_{0}, \cdots$. Each scan cycle is indicated by different color. The positions of the platform at time $t=t_{0}, 2 t_{0}, 3 t_{0}, \cdots$ are also shown in matching color.

Tikhonov regularization and the other based on the total variation regularization. Section 4 presents a group of sensitivity tests and thus provides useful guidelines for possible improvements to the mobile cloud tomography method. Section 5 summarizes the findings of this study.

\section{Description of methodology}

Observation system simulation experiments are useful tools to study a forecast or retrieval system. The observation system simulation for cloud tomography is mainly composed of two components: a forward model to generate virtual cloud tomography observations and an inverse algorithm to retrieve cloud liquid water fields from the simulated radiometric observations. The forward model contains an algorithm for sampling modeled clouds in a manner consistent with microwave radiometer observations. Specifically, the forward model first generates test clouds using a large eddy simulation and then generates virtual microwave observations with prescribed radiometer specifications using a radiative transfer equation. The inverse algorithm numerically solves the radiative transfer equation to obtain the cloud LWC fields.

In order to examine the validity of the mobile cloud tomography method under different environmental conditions, we select two very different cloud cases as the basis for the observation system simulation experiments (Fig. 2). The first case is a mid-latitude stratocumulus cloud simulated by the DHARMA large eddy simulation model driven by data from Atlantic Stratus Experiment (ASTEX) (Ackerman et al., 1995). The stratocumulus clouds in the ASTEX region generally have cloud tops between the 800 and 700 mbar levels. The second case is also a simulation from the same model but is a patchy cumulus situation based on the Atlantic Trade wind Experiment (ATEX) data. The domain where the simulated clouds are located is $5 \mathrm{~km}$ wide and $2.5 \mathrm{~km}$ high.

The two key components of the cloud tomography technique are observing a cloud's microwave emission from multiple directions and locations and reconstructing the spatial

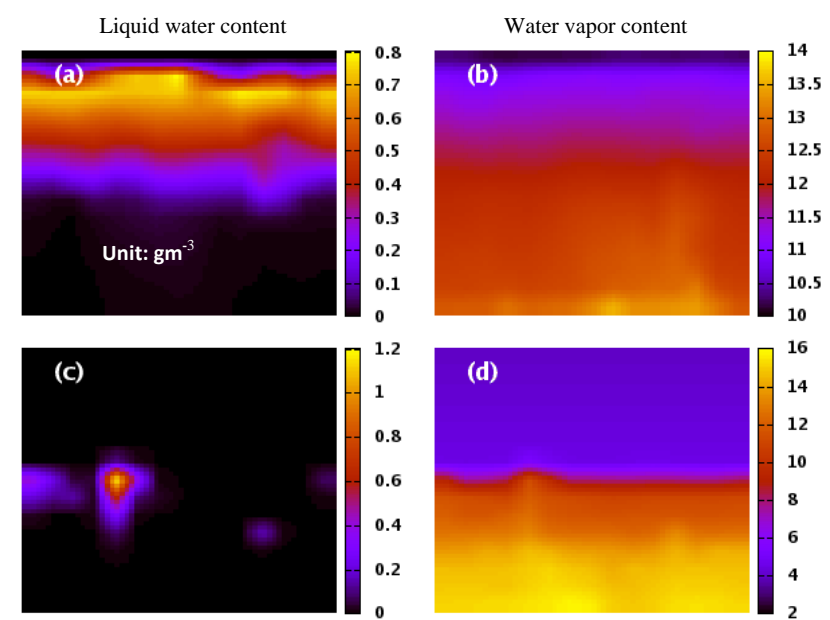

Fig. 2. The cloud liquid water and water vapor fields of a stratocumulus case (ASTEX) and a puffy cumulus case (ATEX) simulated by a large eddy simulation model (Ackerman et al., 1995). (a) Stratocumulus case, liquid water; (b) Stratocumulus case, water vapor; (c) Puffy cumulus case, liquid water; and (d) Puffy cumulus case, water vapor. The domain is $5 \mathrm{~km}$ wide and $2.5 \mathrm{~km}$ high.

distribution of cloud LWC from the resulting observations. The microwave radiation intensity measured by radiometers, usually converted to brightness temperature for convenience, is composed of the path-attenuated emission from the background and the atmospheric emission (microwave emission of clouds, water vapor, and oxygen) along the observation path. By choosing an appropriate working wavelength, such as centimeter waves, the scattering of microwave radiation by cloud droplets and gases in the atmosphere can be neglected. Thus the microwave radiometer observations depend on only the spatial distribution of atmospheric absorption coefficients. They relate to the spatial distribution of cloud LWC and other atmospheric variables through the following radiative transfer equation:

$I\left(\Omega_{i}\right)=I_{\infty} \tau\left(\Omega_{i}, 0, \infty\right)+\int_{0}^{\infty} B(T) \alpha\left(s, \Omega_{i}\right) \tau\left(\Omega_{i}, 0, s\right) d s$,

where $I\left(\Omega_{i}\right)$ is the radiation intensity reaching the radiometer from direction $\Omega_{i} ; I_{\infty}$ is the intensity of the background microwave radiation and $\tau\left(\Omega_{i}, 0, \infty\right)$ indicates the attenuation of the background emission along the path specified by direction $\Omega_{i} ; B(T)$ is the Planck function at temperature $T$; $\alpha$ is the absorption coefficient determined by the atmosphere state; and $\tau\left(\Omega_{i}, s_{1}, s_{2}\right)=\exp \left[-\int_{s_{1}}^{s_{2}} \alpha\left(s, \Omega_{i}\right) d s\right]$ is the transmittance between two points $s_{1}$ and $s_{2}$ on the path along direction $\Omega_{i}$.

A radiometer observation is the convolution of Eq. (1) with the antenna gain pattern $G$ (antenna directivity), which is determined by the size and shape of the antenna and can be partially characterized by antenna beam width. In this study, the antenna gain pattern is assumed to be the widely 
used form in which the gain decreases exponentially with the square of angular departure from the center axis $\Omega_{i}$ (Drake and Warner, 1988),

$$
\begin{aligned}
\bar{I}\left(\Omega_{i}\right) & =\int I(\Omega) G\left(\Omega-\Omega_{i}\right) d \Omega, \\
G(\xi) & =\frac{1}{w}\left(\frac{4 \ln 2}{\pi}\right)^{1 / 2} \exp \left[-4 \ln 2\left(\frac{\xi}{w}\right)^{2}\right] .
\end{aligned}
$$

Here $w$ stands for the width of the antenna beam between rays where the power gain is half its maximum value.

Substituting Eq. (1) into Eq. (2), taking into account the equality $\tau\left(\Omega_{i}, s_{1}, s_{2}\right)=\tau\left(\Omega_{i}, s_{1}, s\right) \tau\left(\Omega_{i}, s, s_{2}\right)$, and approximating the angular integral using Gaussian quadratures, we get:

$$
\begin{aligned}
& \sum_{k=1}^{N_{H}} w_{k} \tau\left(\Omega_{i k}, 0, s_{1}\right) \int_{s_{1}}^{s_{2}} B \alpha \tau\left(\Omega_{i k}, s_{1}, s\right) d s=\bar{I}\left(\Omega_{i}\right) \\
& \quad-\sum_{k=1}^{N_{H}} w_{k} I_{\infty} \tau\left(\Omega_{i k}, 0, \infty\right)-\sum_{k=1}^{N_{H}} w_{k}\left[\int_{0}^{s_{1}}\right. \\
& \left.\quad B \alpha \tau\left(\Omega_{i k}, 0, s\right) d s+\tau\left(\Omega_{i k}, 0, s_{2}\right) \int_{s_{2}}^{\infty} B \alpha \tau\left(\Omega_{i k}, s_{2}, s\right) d s\right] .
\end{aligned}
$$

Here $N_{H}$ is the number of the Gaussian quadrature points; $w_{k}$ is the weight of the antenna gain pattern corresponding to the Gaussian quadrature points; $s_{1}$ and $s_{2}$ are the path lengths from the radiometer to the locations at which the beam with direction $\Omega_{i}$ enters and leaves the cloud.

Given a total number of $m$ beams, Eq. (3) can be further discretized by dividing a domain, which is large enough to contain the clouds of interest, into $n=N^{3}$ ( $N^{2}$ for a 2-D slice) equally-sized pixels to yield the following matrix equation:

$\mathbf{A x}=\boldsymbol{b}$,

where $\boldsymbol{x}^{T}=\left(\alpha_{1}, \alpha_{2}, \cdots, \alpha_{n}\right)$ is the vector of absorption coefficients; $\boldsymbol{b}^{T}=\left(b_{1}, b_{2}, \cdots, b_{m}\right)$, is the vector of adjusted observations, $b_{i}$ equals the right side of Eq. (3) and can be interpreted as the microwave emission from the observation path plus the path-attenuated background emission; and $\mathbf{A}=\left(a_{i j}\right)$ is an $m \times n$ matrix with

$a_{i j}=\sum_{k=1}^{N_{H}} w_{k} \tau\left(\Omega_{i k}, 0, s_{1}\right) \int_{s_{1}}^{s_{2}} B \varphi_{j}\left(s, \Omega_{i k}\right) \tau\left(\Omega_{i k}, s_{1}, s\right) d s$.

$\phi_{j}\left(s, \Omega_{i k}\right)$ is nonzero only if the point $\left(s, \Omega_{i k}\right)$ is in the $j$ th cloud pixel, and there $\phi_{j}=1$.

As shown in Part 1, the tomographic retrieval problem is actually the inversion of the matrix Eq. (4) to obtain the vector of absorption coefficients. In clouds, the absorption coefficient generally consists of contributions from liquid water $\left(\alpha_{l}\right)$, water vapor $\left(\alpha_{v}\right)$, and molecular oxygen $\left(\alpha_{\mathrm{O}_{2}}\right)$. The absorption coefficient is simply a linear function of LWC:

$\alpha=\kappa_{l} \cdot \mathrm{LWC}+\alpha_{v}+\alpha_{\mathrm{O}_{2}}$, where $\kappa_{l}$ is the absorption efficiency of liquid water and depends only on temperature and radiometer frequency. The absorption model for calculating the absorption coefficients for non-precipitating clouds, water vapor, and oxygen are given by Westwater (1972) and Falcone (1966); they are also specified in the Appendix of Warner et al. (1985). The use of more recent absorption model such as that of Rosenkranz (1998) has negligible impacts on the simulation experiments. Our previous studies shows that the retrieval error associated with the uncertainties in humidity and temperature measurements is negligible with current radiosonde techniques. Thus, for simplicity, we assume the distributions of water vapor and atmospheric temperature are known exactly in this study.

To summarize, the observation system simulation experiment for cloud tomography is composed of the following steps: (1) specifying the atmospheric state and cloud fields, choosing appropriate radiometer specifications and data collection strategy (e.g., radiometer scanning speed, speed of platform motion); (2) generating virtual radiometer observations using the radiative transfer equation; (3) inverting the simulated virtual radiometer observations to reconstruct the spatial distribution of microwave absorption coefficient and then calculating the spatial distribution of cloud LWC using Eq. (6).

\section{Comparison of $L_{1}$ and $L_{2}$ regularizations}

As shown in our previous studies, the retrieval problem of cloud tomography, i.e., the inversion of Eq. (4) to obtain vector $\boldsymbol{x}$, is highly ill-posed because of a limited range of viewing angles of clouds (an ideal tomographic reconstruction requires a cloud to be scanned from all directions in a $4 \pi$ sphere centered at the cloud). Regularization techniques that make use of various types of a priori knowledge should be used to obtain physically credible retrievals (Twomey, 1977). As shown in Part 1 of this paper, the regularization of the Eq. (4) in the form of $L p$ norm can be written as,

$$
\begin{aligned}
& \min _{x}\left\{\|\boldsymbol{x}\|_{p}\right\} \text {, subject to }\|\mathbf{A} \boldsymbol{x}-\boldsymbol{b}\|_{2}^{2} \leq \varepsilon \\
& \text { and other constraints. }
\end{aligned}
$$

The notation $\|\cdots\|_{p}$ denotes the $L p$ norm of a vector, and $\varepsilon$ is an error tolerance usually determined by the measurement error and the error associated with the forward radiative transfer model. In this section we examine the skill of two different regularization techniques: the standard Tikhonov regularization that corresponds to $p=2\left(L_{2}\right.$ norm), and the total variation (TV) regularization that corresponds to $p=1\left(L_{1}\right.$ norm).

The $L_{2}$ norm Tikhonov regularization is widely used in many disciplines, and it is relatively easy to implement numerically because of its linearity under differentiation (Hansen, 1998). But the $L_{2}$ norm usually penalizes more when the gradients are large, and thus it tends to bias toward 
a smooth solution (Strong and Chan., 2003). One of our previous studies (Huang et al., 2008b) shows that this method often fails to capture some discontinuous structures around the cloud top where cloud water content decreases from its maximum value to zero in a few tens of meters. Instead, the retrieved cloud top boundaries are often blurred and extended to a higher altitude. Non-linear regularization techniques such as the $L_{1}$ norm TV regularization are also becoming more and more popular. The main advantage of the TV regularization is that it does not penalize discontinuities in the solution, while simultaneously not penalizing smoothness in the solution; thus under certain conditions it can preserve the discontinuous structures in the solution (Acar and Vogel, 1994; Chambolle and Lions, 1997). Let us use a simple example presented in Pederson (2005) to illustrate the point. Inspecting the piecewise linear function illustrated in Fig. 3, we can calculate the $L p$ norm (raised to the power $p$ ) of the gradient of $f(t)$ as follows:

$$
\begin{aligned}
& \left\|f^{\prime}(t)\right\|_{p}^{p}=\int_{-\infty}^{+\infty}\left|f^{\prime}(t)\right|^{p} d t \\
& =\int_{0}^{h}\left(\frac{d}{h}\right)^{p} d t \\
& =d^{p} h^{1-p}
\end{aligned}
$$

Hence for $p=1$ (the TV regularization), we have

$\left\|f^{\prime}(t)\right\|_{1}=d$.

And for $p=2$ (the standard Tikhonov regularization),

$$
\left\|f^{\prime}(t)\right\|_{2}^{2}=d^{2} h^{-1} \text {. }
$$

This means that if $p=1$ the width of the interval $h$ has no influence on the regularization term; thus the TV regularization should have no bias that depends on the scale of the structure. On the other hand, for $p=2$ it is easy to verify that the smaller $h$ is, the larger the regularization term becomes. Therefore the $L_{2}$ norm Tikhonov method tends to suppress large discontinuities at small scales and is likely to miss highfrequency discontinuous structures (Strong and Chan, 2003). However, the TV regularization is difficult to implement numerically and also computationally expensive because of its nonlinearity under differentiation.

The linear Tikhonov method can be implemented in a direct manner by calculating the inverse of the regularized kernel matrix, but such direct techniques require a large amount of computer memory and are also computationally expensive (Hansen, 1998). Both the Tikhonov and the TV regularization problems can also be solved in an iterative manner, using techniques such as the widely used algebraic reconstruction technique (Gordon et al., 1970; Twomey, 1987). In Part 1 of this paper, we present an iterative algorithm that can determine the weight of the regularization term adaptively. Here we carry out a test to verify whether the iterative retrieval algorithm presented in Part 1 of this paper yields the same solution as the direct inversion method. The reference cloud

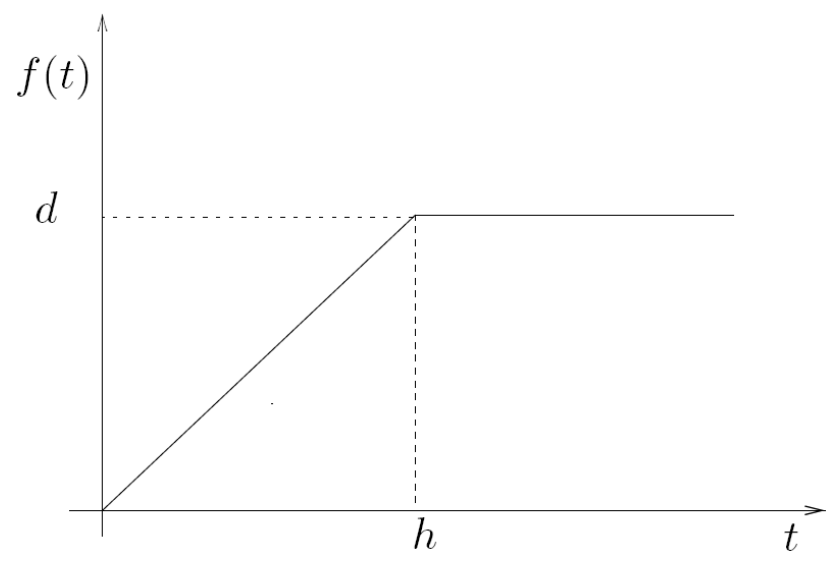

Fig. 3. A piecewise linear function used to illustrate the difference between the $L_{1}$ norm total variation regularization and the $L_{2}$ norm Tikhonov regularization.

cases are the stratocumulus and broken cumulus clouds described in Sect. 2 (Fig. 2). A virtual airborne cloud tomography setup is used to generate the simulated tomographic observations. More details on the specifications of the airborne tomography setup can be found in Sect. 3. The direct algorithm described in Huang et al. (2008b) and the iterative retrieval algorithm described in Part 1 are then used to invert the simulated tomographic observations to obtain retrieved cloud LWC fields. The difference between the results of the two retrieval algorithms is negligible for both cloud cases. In the rest of our observation system simulation experiments, we use the iterative algorithm because it does not require multiple retrieval runs to determine the optimal weighting parameter for the regularization.

We then conduct four observation system simulation experiments for the airborne cloud tomography setup using the two cloud cases and the two regularization techniques. Figure 4 shows the retrieved cloud LWC fields using the standard Tikhonov regularization and the TV regularization for the two cloud cases. The rms error of the Tikhonov retrieval is $0.09 \mathrm{gm}^{-3}$ for the stratocumulus cloud case (Fig. 4a) and $0.03 \mathrm{gm}^{-3}$ for the broken cumulus case (Fig. $4 \mathrm{~b}$ ). For both cloud cases, the Tikhonov retrievals reproduce the location and shape of clouds with reasonable fidelity. As expected, the Tikhonov method appears to smooth the sharp cloud top edges where the largest discontinuity of cloud LWC is located. As a result, the retrieved cloud top is extended to a much higher altitude for both cloud cases. Also some patchy clouds with very low water content appear in some regions that are clear sky in the reference images, possibly associated with the random noises added to the simulated radiometric observations. When the TV regularization is used, the retrieval error for both cloud cases is reduced significantly; it becomes $0.065 \mathrm{gm}^{-3}$ for the stratocumulus (Fig. 4c) and $0.02 \mathrm{gm}^{-3}$ for the broken cumulus (Fig. $4 \mathrm{~d}$ ). The significant 

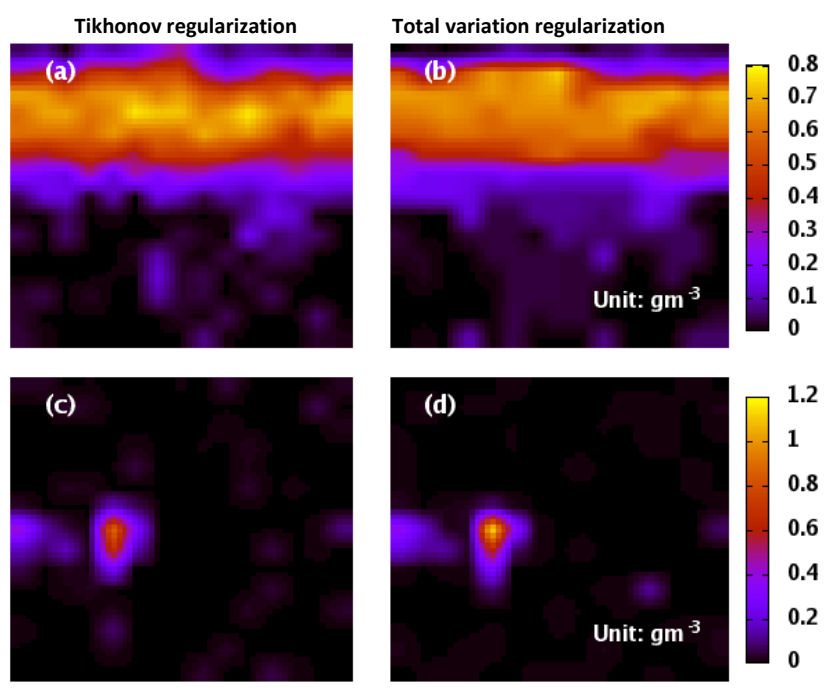

Fig. 4. The cloud liquid water fields retrieved using the airborne cloud tomography setup with the total variation regularization and the standard Tikhonov regularization. (a) stratocumulus case using the Tikhonov regularization; (b) stratocumulus case using the total variation regularization; (c) puffy cumulus case using the Tikhonov regularization; and (d) puffy cumulus case using the total variation regularization.

reduction in retrieval error indicates that the $L_{1}$ norm TV regularization is superior to the $L_{2}$ norm Tikhonov regularization for the ill-posed cloud tomography problem. The cloud LWC fields are reproduced with higher fidelity in the TV regularization retrievals than in the Tikhonov retrievals. The sharp cloud top edges now are well reproduced in the TV regularization retrievals, clearly showing the superior skill of the TV regularization in preserving discontinuous structures.

\section{Results of sensitivity studies}

In this section, we conduct a series of observation system simulation experiments to investigate the effects of a variety of factors on the tomographic retrieval and to determine the optimal tomographic configuration and data acquisition strategies. The factors examined include radiometer characteristics, radiometer scan strategy, background microwave characteristics, and platform speed and height.

\subsection{Ground-based and airborne setups}

There are several important differences between ground- and aircraft-based cloud tomography setups. Among them are speed of platform motion relative to clouds and background microwave brightness temperature. For a ground-based setup the speed of a truck trailer is typically on the order of $10 \mathrm{~m} / \mathrm{s}$, while the speed of an aircraft is usually one order of magnitude faster than that of a truck. The large difference in platform speed can result in very different data geometries and thus can impact the retrieval accuracy in a significant way. Another major difference between the two setups is that the ground radiometer scan clouds from below while the airborne radiometer usually scans clouds from above due to the limitation of aircraft design. Thus the microwave contrast between clouds and the background will be very different for these two setups. For the ground-based setup, the atmospheric background emission consists of the cosmic background emission plus the gaseous emission from the atmosphere above cloud tops. The cosmic background is well known to be around $2.7 \mathrm{~K}$ and is in sharp contrast with clouds. For the aircraft-based setup, the background is either land surface or sea surface and the brightness temperature ranges from 150 to $250 \mathrm{~K}$ in the microwave Ka band, which is more similar to microwave emission from clouds. In this research we consider only sea surface backgrounds in the airborne tomography simulations because land surfaces have high emissivity in the microwave region and thus have very low contrast with warm clouds. The directional variation of sea surface microwave emission is simulated by a two-scale thermal emission model (Johnson, 2006).

Table 1 shows the typical values of radiometer and platform specifications of the two tomographic setups; these values are used throughout the sensitivity studies if not mentioned otherwise. The radiometer is assumed to have $0.5 \mathrm{~K}$ random noise and $2.3^{\circ}$ beam width. The duration of each radiometer scan cycle is $43 \mathrm{~s}$ and only the beams within $80^{\circ}$ from the nadir or zenith are considered as a valid observation (ground and water vapor emission could overwhelm the radiometer observation if the beam were too close to the horizon). The atmospheric background brightness temperature is $20 \mathrm{~K}$ for the ground-based configuration, and a $0.5 \mathrm{~K}$ random noise is also added in all simulation experiments. For the airborne configuration, the background brightness strongly depends on the incidence angle and is simulated by a two-scale sea surface thermal emission model by assuming a $283 \mathrm{~K}$ surface temperature. A $0.5 \mathrm{~K}$ random noise is also added to the sea surface brightness temperature. The speed of motion of the ground-based platform is assumed to be $24 \mathrm{~m} / \mathrm{s}$, while the aircraft is assumed to fly at a speed of $96 \mathrm{~m} / \mathrm{s}$ at an altitude of $3500 \mathrm{~m}$.

We perform a number of system simulation experiments for each of the two mobile tomography setups using the two cloud cases described in Sect. 2. The radiometer specifications are identical for the ground-based and airborne setups but the background brightness temperature, platform altitude, and speed of platform motion are different. Figures 5 and 6 show the cloud LWC fields retrieved using the two tomography setups along with the corresponding retrieval error. For the stratocumulus cloud case in Fig. 5, the retrieval error of the airborne setup $\left(0.061 \mathrm{gm}^{-3}\right)$ is $50 \%$ higher than that of the ground-based setup $\left(0.041 \mathrm{gm}^{-3}\right)$. The groundbased retrieval accurately reproduces the base and top of the stratocumulus clouds, while the retrieval from the airborne setup shows more spurious patchy clouds in clear sky regions 
Table 1. The typical radiometer specifications, background characteristics, and platform parameters of the ground-based and airborne cloud tomography configurations.

\begin{tabular}{lccccccccc}
\hline & \multicolumn{4}{c}{ Radiometer characteristics } & \multicolumn{3}{c}{ background } & platform \\
\cline { 2 - 9 } & $\begin{array}{c}\text { noise, } \\
\mathrm{K}\end{array}$ & $\begin{array}{c}\text { frequency, } \\
\mathrm{GHz}\end{array}$ & $\begin{array}{c}\text { beam width, } \\
\text { degree }\end{array}$ & $\begin{array}{c}\text { scan period, } \\
\mathrm{s}\end{array}$ & $\begin{array}{c}\text { Integration } \\
\text { time, } \mathrm{s}\end{array}$ & $\begin{array}{c}\text { brightness } \\
\text { temperature, } \mathrm{K}\end{array}$ & $\begin{array}{c}\text { uncertainty, } \\
\mathrm{K}\end{array}$ & $\begin{array}{c}\text { speed, } \\
\mathrm{m} / \mathrm{s}\end{array}$ & $\begin{array}{c}\text { altitude, } \\
\mathrm{km}\end{array}$ \\
\hline Ground-based & 0.5 & 31.6 & 2.3 & 43 & 0.3 & 20 & 0.5 & 24 & 0 \\
Airborne & 0.5 & 31.6 & 2.3 & 43 & 0.3 & $165-180$ & 0.5 & 96 & 3.5 \\
\hline
\end{tabular}
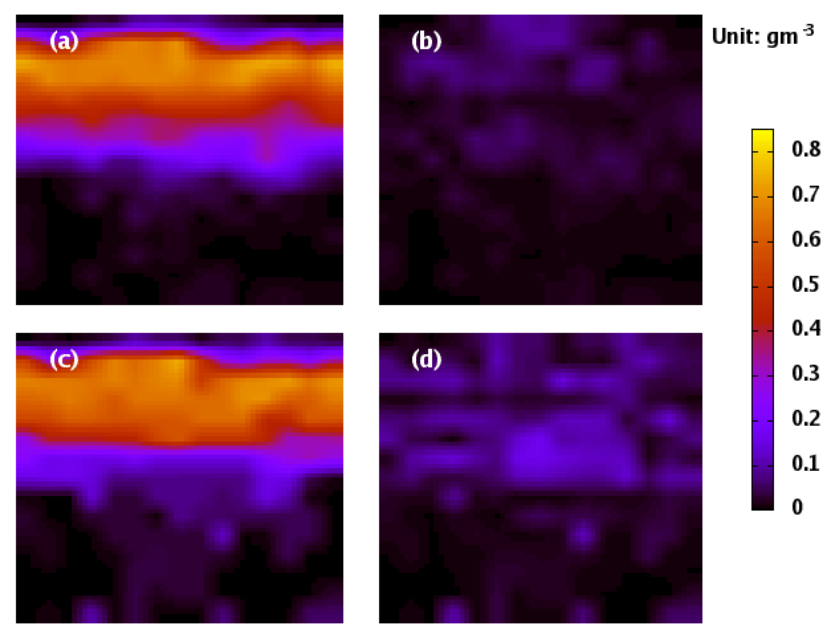

Fig. 5. The stratocumulus clouds retrieved using the ground-based and airborne setups, as well as the corresponding images of error. (a) Retrieved liquid water field by the ground-based setup; (b) the error of the ground-based retrieval; (c) retrieved liquid water field by the airborne setup; (d) the error of the airborne retrieval.

(Fig. 5). For the patchy cumulus case (Fig. 6), the result is very similar. The retrieval error of the ground-based setup is $0.006 \mathrm{gm}^{-3}$, substantially lower than that of the airborne setup $\left(0.02 \mathrm{gm}^{-3}\right)$. The airborne retrieval shows some puffy clouds with low water content in several clear sky regions, while the ground-based retrieval reproduces the original image with such high fidelity that one can hardly tell any difference from the reference image (Fig. 6). The reason why the ground-based setup is superior to the aircraft-based setup will be discussed in Sects. 4.3 and 4.4.

\subsection{Radiometer noise level}

The second factor examined is the radiometer noise level. The experiment is based on the stratocumulus cloud case. Figure 7 shows that the retrieval error for both the groundbased and airborne setups increases monotonically with the radiometer noise level, as one would expect. The retrieval error of the ground-based setup increases by a factor of 3 from 0.036 to $0.12 \mathrm{gm}^{-3}$ when the radiometer noise level in-
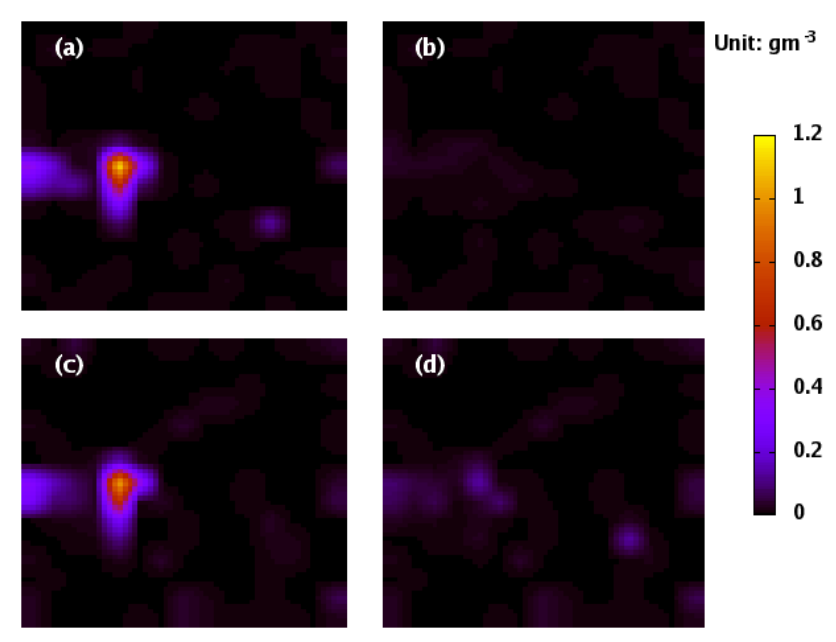

Fig. 6. The puffy cumulus clouds retrieved using the ground-based and airborne setups, as well as the corresponding images of error. (a) Retrieved liquid water field by the ground-based setup; (b) the error of the ground-based retrieval; (c) retrieved liquid water field by the airborne setup; (d) the error of the airborne retrieval.

creases from 0.1 to $6.0 \mathrm{~K}$. And for the airborne setup, the retrieval error increases from 0.06 to $0.15 \mathrm{gm}^{-3}$. The retrieval error increases at a slower rate when the noise level is high $(>2.0 \mathrm{~K})$.

The noise level of modern microwave radiometers is typically in the range of 0.1 to $0.5 \mathrm{~K}$. According to Fig. 7, the tomographic retrievals based on such radiometers are accurate to within $5 \%$ of the maximum cloud water content. Even when the noise is extremely high $(>4 \mathrm{~K})$, the simulations show that the tomographic retrievals can still roughly reproduce some of the cloud features but high-frequency structures are difficult to resolve because the retrievals tend to rely more on the arbitrary mathematical constraints rather than the observations.

\subsection{Uncertainty in the estimate of the background brightness temperature}

The third factor considered in this research is the uncertainty in the estimate of background radiometric characteristics, i.e., the atmospheric background brightness temperature for 


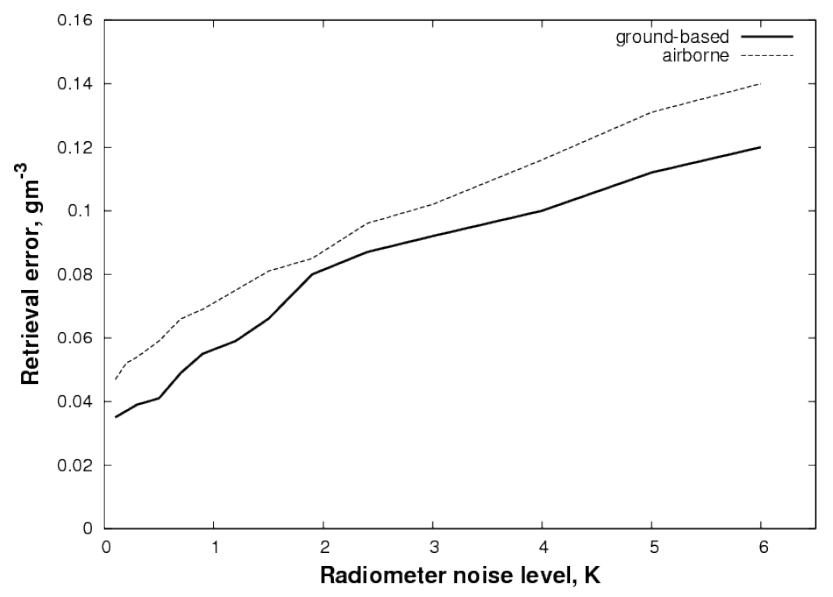

Fig. 7. The retrieval error as a function of radiometer noise level. As expected, the retrieval error increases with radiometer noise level for both the ground-based and airborne setups. The stratocumulus cloud case is used for this sensitivity test.

the ground-based setup and the sea surface brightness temperature for the airborne setup. For the ground-based setup, the cosmic background is well-known to be around $2.7 \mathrm{~K}$, but the water vapor emission between the cloud top and the top of the atmosphere also contributes to the radiometer observations and thus should be considered as a part of background emission. Accounting for this, we set the mean background brightness temperature of the ground-based setup to be $20 \mathrm{~K}$ and add varying levels of random error (from 0.1 to $6 \mathrm{~K}$ ) to the background. The sea surface microwave emission is a function of incidence angle and the angular distribution is determined by surface roughness and the orientation of sea waves (Johnson, 2006). For example, wind-driven waves and foam can lead to an uncertainty much larger than $3 \mathrm{~K}$ in the simulation of directional brightness temperatures. Figure 8 shows that, similar to the noise in the radiometric observations, the uncertainty in the background brightness temperature also has significant effects on the retrieval accuracy. The retrieval error increases with background uncertainty, while the rate of this increase decreases with the background uncertainty.

The microwave contrast between the atmospheric background and clouds is much greater than the contrast between the sea surface and clouds. Thus the signal-to-noise ratio of the ground-based setup will be higher than that of the airborne setup if the radiometer noise level is the same. Furthermore, wind-driven sea surface waves and foam can add substantial uncertainty to the observing or modeling of the sea surface brightness temperature. As a result, the observing or modeling error of the sea surface brightness temperature is usually much higher than that of the atmospheric background. Therefore, the higher retrieval accuracy of the ground-based setup can be partly attributed to the differences in the characteristics of the background emission.

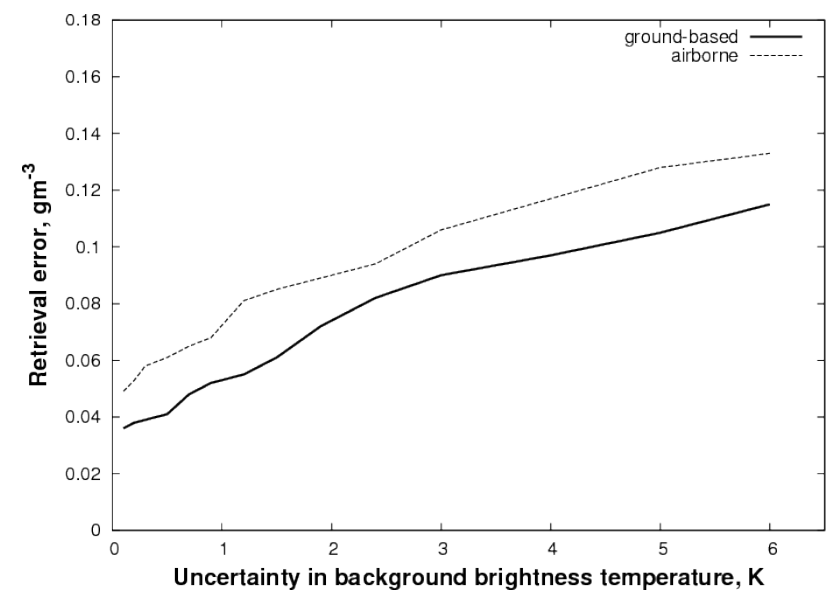

Fig. 8. The retrieval error increases with the uncertainty in the estimate of background brightness temperature for both the groundbased and airborne setups. The stratocumulus cloud case is used for this sensitivity test.

\subsection{Scan strategy}

Our previous studies (Huang et al., 2008a, b) have shown that beam intersection is crucial to the success of a tomographic reconstruction. If successive scans do not overlap with each other, one will be able to retrieve only the pathintegrated cloud water content. The duration of each radiometer scan cycle (proportional to the inverse of radiometer scanning speed) is important to the geometry of the tomographic data; it is a key factor that determines to what extent the microwave beams from successive scan cycles intersect with each other.

Figure 9 illustrates that the retrieval error increases monotonically with the duration of radiometer scan cycle. For the ground-based setup, the retrieval error varies by a factor of 4 from 0.032 to $0.13 \mathrm{gm}^{-3}$ when the duration of radiometer scan cycle changes from 10 to $160 \mathrm{~s}$. For the airborne setup, the retrieval error increases by a factor of 3 from 0.045 to $0.14 \mathrm{gm}^{-3}$ with the same change in the duration of radiometer scan cycle. This result can be explained by two facts. First, there will be more scan cycles if the radiometer scans faster since the total time needed to pass the clouds of interest depends only on the speed of platform motion. Second, with a faster scanning radiometer, the distance between two successive cycles will be smaller and thus the swathes of these scan cycles will have more overlap with each other. Therefore, from the geometric perspective, fast scanning radiometers are preferred in tomographic applications. On the other hand, a faster scan means shorter integration time for each beam and thus greater random noise in data, which will have an adverse effect on the retrieval accuracy. The optimal choice of radiometer scanning speed can be found by taking both factors into account. 


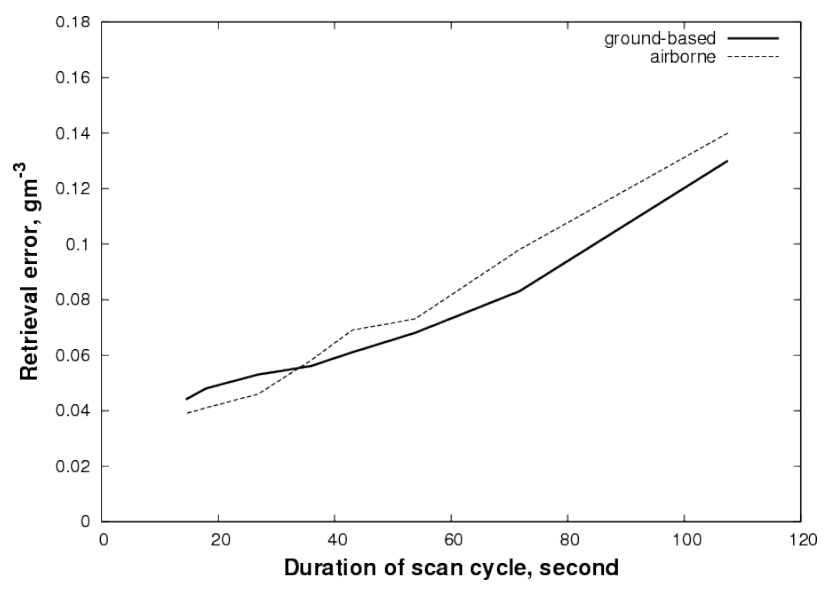

Fig. 9. The retrieval error as a function of the duration of radiometer scan cycle (proportional to the inverse of radiometer scanning speed). For both the ground-based and airborne setups, a faster radiometer results in more accurate tomographic retrievals. The stratocumulus cloud case is used for this sensitivity test.

\subsection{Speed of platform motion}

Speed of platform motion, similar to radiometer scanning speed, is a key factor that determines how many scans the radiometers can perform while the platform passes the clouds of interest and determines the distance between successive scans. If the platform moves so fast that successive scans are well separated and do not overlap with each other, it will be difficult to reconstruct the cloud structure from these tomographic observations. As illustrated in Fig. 10, a reduced platform speed substantially improves the retrieval accuracy for both the ground-based and airborne setups. For the ground-based setup, the retrieval error increases from 0.036 to $0.073 \mathrm{gm}^{-3}$ when the platform speed increases from 24 to $96 \mathrm{~m} / \mathrm{s}$. For the airborne setup, we vary the platform speed from 64 to $240 \mathrm{~m} / \mathrm{s}$. Similarly, the retrieval error increases with the aircraft speed, changing from 0.049 to $0.12 \mathrm{gm}^{-3}$. When the platform moves slowly, the swath of each scan cycle will overlap significantly with its successive scans (and also the previous scans) and thus there will be more valuable information in the tomographic observations for retrieving cloud structure. When the platform speed exceeds $200 \mathrm{~m} / \mathrm{s}$ (each scan cycle at best overlaps with its previous and successive scan cycles), the tomographic retrieval will become very difficult because of the very limited beam intersection.

The typical speed of a research aircraft is $100-300 \mathrm{~m} / \mathrm{s}$, almost one order of magnitude faster than that of a groundbased platform. This provides another explanation for the results in Sect. 4.1 that the ground-based setup substantially outperforms the airborne setup.

However, a slower moving platform means that it will take a longer time to collect the necessary data. The life time of some types of clouds is on the order of tens of minutes and

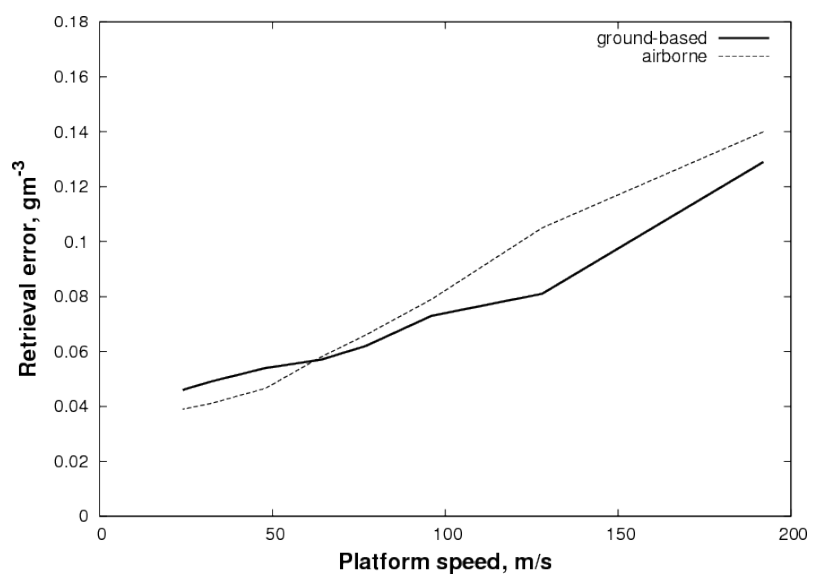

Fig. 10. The retrieval error as a function of the speed of platform motion. A slower platform provides more accurate retrieval at the expense of longer data collection time and possible loss of ability to monitor rapidly-evolving clouds. The stratocumulus cloud case is used for this sensitivity test.

clouds can evolve significantly during the period when the tomographic data are collected. Practically, there is always a trade-off between choosing a slow speed of platform motion and minimizing the impacts of cloud evolution during data collection.

\subsection{Platform altitude}

The geometry of tomographic observations, specifically the range of radiometer viewing angles (indicated by $\Omega$ in Fig. 11), often plays an important role in determining the total amount of useful information in the tomographic data. In general, the larger the angle $\Omega$ spanned by the radiometer beams (those hitting the cloud), the more useful information in the resultant observations. An ideal tomographic reconstruction requires the target to be viewed from all directions, i.e., the range of viewing angles should be $360^{\circ}$. In cloud tomography applications, the range of viewing angles $\Omega$ is usually much smaller than $180^{\circ}$ and as a consequence the retrieval problem becomes highly ill-posed. The range of viewing angles is often limited by the platform altitude as shown in Fig. 11. If the aircraft flies at an altitude that is much higher than the cloud top, it will need to move a large distance in order to cover a useful range of viewing angles, and it will take a longer time to collect the tomographic data.

Figure 12 shows that the retrieval error increases when the aircraft altitude increases from 2.5 to $8 \mathrm{~km}$ (the cloud top is about $2.2 \mathrm{~km}$ ). This result is consistent with the fact that the range of viewing angles decreases with platform altitude if the total observation time (thus the distance the aircraft travels) is fixed. The retrieval error increases by a factor of two from 0.07 to $0.13 \mathrm{gm}^{-3}$ when the aircraft altitude changes from 2.5 to $7 \mathrm{~km}$. The optimal aircraft altitude seems to be 


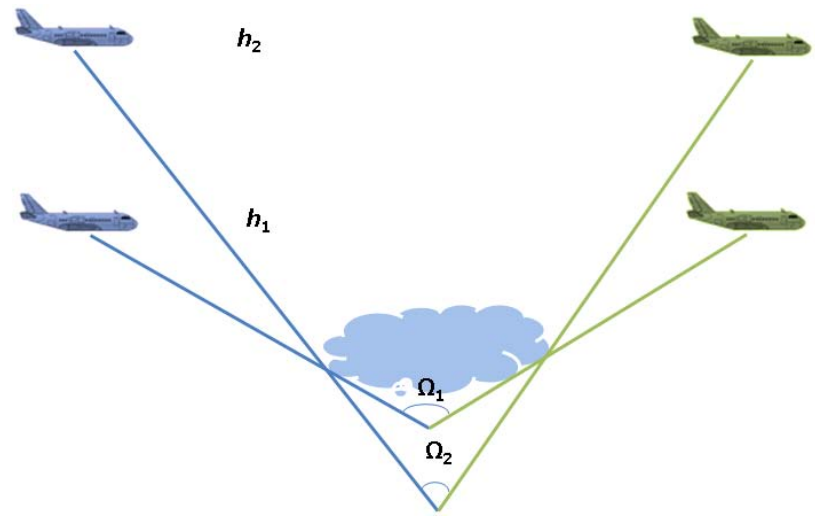

Fig. 11. Illustration of the dependence of radiometer viewing angle on the aircraft altitude. The range of radiometer viewing angles (indicated as $\Omega_{1}, \Omega_{2}$ ) decreases with the vertical distance between the aircraft and the cloud.

between 2.8 and $3.5 \mathrm{~km}$. If the aircraft is above $5.5 \mathrm{~km}$, the retrieval of the airborne setup can hardly reproduce the cloud top boundary, indicating the lack of information in the tomographic observations. When the aircraft is close to the cloud top (between 2.8 and $4.5 \mathrm{~km}$ ), both the cloud base and top boundaries can be reproduced accurately. But if the aircraft is too close to the cloud top, the scan cycles will have little or no overlap around the cloud top; so the tomographic observations again will not contain enough information to retrieve cloud structures around the cloud top.

\section{Conclusions}

Part 1 of this research examined the results of a very limited cloud tomography experiment during the 2003 AMSR-E validation campaign at Wakasa Bay and demonstrated that this tomographic method is capable of retrieving spatial distributions of cloud liquid water using only a single scanning microwave radiometer aboard a research aircraft. It was found that during the experiment many conditions were not optimal for cloud tomography retrieval, such as high aircraft altitude, fast speed of aircraft motion, and strong surface wind (thus large uncertainty in the estimate of sea surface thermal emission). Part 2 (this paper) then focuses on a sensitivity study using observation system simulation experiments to investigate the factors that determine the accuracy of tomographic retrievals.

We conducted a series of observation system simulation experiments both by varying the specifications of the cloud tomography configuration and by choosing different inversion techniques. Two inversion techniques were examined: the first one based on the Tikhonov regularization $\left(L_{2}\right.$ norm) and the other based on the total variation regularization $\left(L_{1}\right.$ norm). The retrieved cloud LWC from the Tikhonov regularization method is accurate to within $10 \%$ of the maxi-

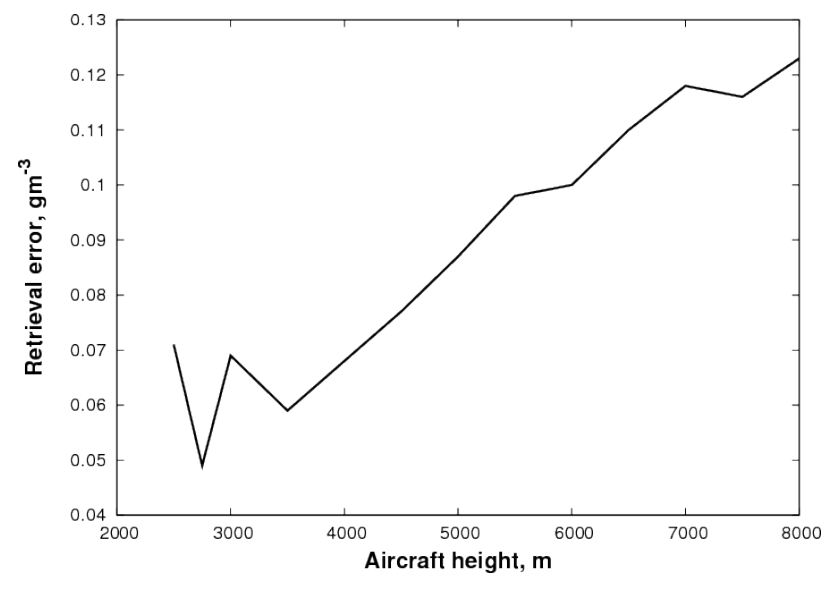

Fig. 12. The retrieval error of the airborne setup as a function of aircraft altitude. The altitude of the cloud top is about $2200 \mathrm{~m}$. The retrieval error first decreases with aircraft altitude, reaches a minimum when the aircraft is $500-1500 \mathrm{~m}$ higher than the cloud top, and then increases with further increase of aircraft altitude. The stratocumulus cloud case is used for this sensitivity test.

mum LWC value in the cloud and captures many features of the cloud field in the original image. Nevertheless, the retrieved cloud top boundary appears to be much smoother than that in the original image; this concurs with the results from our previous studies that the $L_{2}$ norm regularization can hardly preserve large discontinuities. The retrieval from the $L_{1}$ norm total variation regularization shows some noticeable improvements over its $L_{2}$ norm counterpart: the retrieval error is significantly reduced, the sharp cloud edges are well captured, and cloud morphology is also reproduced with high fidelity.

Our observation system simulation experiments show that, besides the choice of inversion technique, the retrieval accuracy also depends on many other factors such as radiometer characteristics, scanning strategy, platform speed, and platform altitude. A slow-moving ground-based cloud tomography setup substantially outperforms a typical airborne setup in terms of retrieval accuracy. There are two reasons for this: (1) the slower ground-based setup allows for a longer cloud observation time (thus more scan cycles) and more scan-to-scan beam overlap than the airborne one; (2) the microwave brightness temperature of clouds contrasts more sharply with the atmospheric background than with the sea surface (thus the ground-based setup tends to have higher signal-to-noise ratio). It is found that the retrieval error increases with the radiometer noise level and the uncertainty in the estimate of background brightness temperature. The radiometer scan speed is another factor that plays an important role in determining the tomographic retrieval quality. Fast scanning means more scan cycles and more overlap between the swaths of successive scan cycles if other conditions are the same, and thus it will lead to improved retrieval accuracy. 
The speed of platform motion is also a critical parameter to cloud tomography observations. A slow platform allows for more scanning data and more overlap between the swaths of the radiometer scan cycles. Thus, in this respect, a slow platform is favorable to cloud tomography. On the other hand, clouds evolve at the same time as the radiometric observations are taken and thus a fast platform has the advantage of being able to capture fast evolution of the clouds. The last parameter examined is the altitude of the aircraft. The simulation experiments show that the optimal aircraft altitude is about 0.5 to $1 \mathrm{~km}$ above the cloud top. When the distance between the aircraft and the cloud top becomes comparable to the horizontal dimension of the cloud, the range of radiometer viewing angles will be limited, and thus accurate retrieval of cloud structures will be difficult.

Acknowledgements. This work has been supported by the DOE Atmosphere Radiation Measurement program under Contract DE-AC02-98CH10886. We thank Emil Sidky, Yangang Liu, and Michael Jensen for many insightful discussions that help to improve this study. We thank two reviewers for constructive comments that improved the manuscript.

Edited by: W. E. Asher

\section{References}

Acar, R. and Vogel, C. R.: Analysis of total variation penalty methods, Inv. Prob., 10, 1217-1229, 1994.

Ackerman, S. A., Toon, O. B., and Hobbs, P. V.: A model for particle microphysics, turbulent mixing, and radiative transfer in the stratocumulus topped marine boundary layer and comparisons with measurements, J. Atmos. Sci., 52, 1204-1236, 1995.

Chambolle, A. and Lions, P. L.: Image recovery via total variation minimization and related problems, Numer. Math., 72, 167-188, 1997.

Drake, J. F. and Warner, J.: A theoretical study of the accuracy of tomographic retrieval of cloud liquid with an airborne radiometer, J. Atmos. Sci. Ocean. Tech., 5, 844-857, 1988.

Falcone, V.: Calculation of apparent sky temperature at millimeter wavelengths, Radio Sci., 1 (new series), 1205-1209, 1966.

Frisch, A. S., Fairall, C. W., and Snyder, J. B.: Measurement of stratus cloud and drizzle parameters in ASTEX with a Ka-band Doppler radar and a microwave radiometer, J. Atmos. Sci., 52, 2788-2799, 1995.

Gordon, R., Bender, R., and Herman, G. T.: Algebraic Reconstruction Techniques (ART) for Three-dimensional Electron Microscopy and X-ray Photography, J. Theor. Biol., 29, 471-481, 1970.

Hansen, P. C.: Rank deficient and ill-posed problems: Numerical aspects of linear inversion, SIAM, Philadelphia, 247 pp., 1998.

Hogan, R. J., Gaussiat, N., and Illingworth, A. J.: Stratocumulus liquid water content from dual-wavelength radar, J. Atmos. Ocean. Tech., 22, 1207-1218, 2005.
Huang, D., Liu, Y., and Wiscombe, W.: Determination of cloud liquid water distribution using 3D cloud tomography, J. Geophys. Res., 113, D13201, doi:10.1029/2007JD009133, 2008a.

Huang, D., Liu, Y., and Wiscombe, W.: Cloud tomography: Role of constraints and a new algorithm, J. Geophys. Res., 113, D23203, doi:10.1029/2008JD009952, 2008b.

Huang, D., Gasiewski, A. J., and Wiscombe, W.: Tomographic retrieval of cloud liquid water fields from a single scanning microwave radiometer aboard a moving platform - Part 1: Field trial results from the Wakasa Bay experiment, Atmos. Chem. Phys., 10, 6685-6697, doi:10.5194/acp-10-6685-2010, 2010.

Johnson, J. T.: An efficient two-scale model for the computation of thermal emission and atmospheric reflection from the sea surface, IEEE T. Geosci. Remote., 44, 560-568, 2006.

Liljegren, J., Clothiaux, E., Mace, G., Kato, S., and Dong, X.: A new retrieval for cloud liquid water path using a ground-based microwave radiometer and measurements of cloud temperature, J. Geophys. Res., 106(14), 485-500, 2001.

Lobl, S. E., Aonashi, K., Griffith, B., Kummerow, C., Liu, G., Murakami, M., and Wilheit, T.: Wakasa Bay - An AMSR Precipitation Validation Campaign, Bu. Am. Meteorol. Soc., 88, 551-558, 2007.

Pederson, J.: Modular Algorithms for Large-Scale Total Variation Image Deblurring. Technical University of Denmark, master thesis, 122 pp., 2005.

Rosenkranz, P. W.: Water vapor microwave continuum absorption: A comparison of measurements and models, Radio Sci., 33(4), 919-928, doi:10.1029/98RS01182, 1998.

Strong, D. and Chan, T. F.: Edge-preserving and scale-dependent properties of the total variation regularization, Inverse Problems, 19, 165-187, 2003.

Stephens, G. L.: Cloud feedbacks in the climate system: a critical review, J. Climate, 18, 237-273, 2005.

Twomey, S.: Introduction to the mathematics of inversion in remote sensing inversion and indirect measurements, Elsevier, Amsterdam, 243 pp., 1977.

Twomey, S.: Iterative nonlinear inversion methods for tomographic problems, J. Atoms. Sci., 44, 3544-3551, 1987.

Warner, J., Drake, J. F., and Krehbiel, P. R.: Determination of cloud liquid water distribution by inversion of radiometric data, J. Atmos. Ocean. Tech., 2, 293-303, 1985.

Warner, J., Drake, J. F., and Snider, J. B.: Liquid water distribution obtained from coplanar scanning radiometers, J. Atmos. Ocean. Tech., 3, 542-546, 1986.

Warner, J. and Drake, J. F.: Field tests of an airborne remote sensing technique for measuring the distribution of liquid water in convective cloud, J. Atmos. Ocean. Tech., 5, 833-843, 1988.

Weare, B. C.: Evaluation of the vertical structure of zonallyaveraged cloudiness and its variability in the Atmospheric Model Intercomparison Project, J. Climate, 9, 3419-3431, 1996.

Westwater, E. R.: Microwave emission from clouds, NOAA Tech. Rep. ERL 219-WPL, 18, 43 pp., 1972.

Westwater, E. R., Crewell, S., and Matzler, C.: A review of surface based microwave and millimeter wave radiometric remote sensing of the troposphere, Radio Sci. Bull., 3010, 59-80, 2004. 